\title{
Nécessité d'un anion exogène mobile en cas de nutrition ammoniacale
}

\author{
A. Soltani ${ }^{1}$, M. Hajji ${ }^{1}$ et C. Grignon ${ }^{2}$ \\ 1 Institut National de Recherches Scientifiques et Techniques, Borj Cedria, B.P. 95, 2050 Hammam Lif, Tunisie; \\ 2 ENSA-INRA, biochimie et physiologie végétales (CNRS URA 573), 34060 Montpellier Cedex 1, France
}

(reçu le 24 février 1989, accepté le 16 mai 1989)

\begin{abstract}
Résumé - Des plantules d'orge âgées de 6 j sont cultivées pendant 1 mois sur des milieux contenant $\mathrm{NO}_{3}^{-}$ou $\mathrm{NH}_{4}^{+}$ comme source de $\mathrm{N}$. Des variantes de ces milieux permettent de comparer les effets de la mobilité de l'anion qui accompagne $\mathrm{NH}_{4}^{+}\left(\mathrm{Cl}^{-}\right.$, facilement absorbé, ou $\mathrm{SO}_{4}^{2-}$, peu mobile). La réduction de croissance, classiquement décrite lorsque $\mathrm{NH}_{4}^{+}$est substitué à $\mathrm{NO}_{3}^{-}$, apparaît seulement quand l'anion majeur est $\mathrm{SO}_{4}^{2-}$. Avec $\mathrm{Cl}^{-}$, les 2 sources d'azote permettent des croissances semblables. Dans le cas de $\mathrm{NO}_{3}^{-}$, les acides organiques (évalués par la différence entre les cations et les anions exogènes dosés dans les tissus) représentent plus de la moitié des anions accumulés. Sur milieu $\mathrm{NH}_{4}^{+}$, la somme des anions exogènes équilibre celle des cations. Quand le milieu $\mathrm{NH}_{4}^{+}$ne contient que les anions peu mobiles $\mathrm{SO}_{4}^{2-}$ et $\mathrm{H}_{2} \mathrm{PO}_{4}^{-}$, l'accumulation ionique totale reste très inférieure à celle qui est observée en présence de $\mathrm{Cl}^{-}$. La réduction de croissance qui est alors observée semble due à l'incapacité des plantes à accumuler les anions exogènes, dans une situation où la production nette d'anions organiques est interdite par l'assimilation de $\mathrm{NH}_{4}^{+}$.
\end{abstract}

orge - nitrate - accumulation ionique - croissance - échanges de $\mathrm{H}^{+}$

Summary - Ammonia nutrition and the need for a mobile exogenous anion. Six-day-old barley seedlings were grown for 1 month in media containing eigher $\mathrm{NO}_{3}^{-}$or $\mathrm{NH}_{4}^{+}$as $\mathrm{N}$ source. The effects of the anion which accompanied $\mathrm{NH}_{4}^{+}$were studied $\left(\mathrm{Cl}^{-}\right.$or $\mathrm{SO}_{4}^{2-}$ readily, respectively poorly, absorbed by barley). The growth reduction, which is classically observed when $\mathrm{NO}_{3}^{-}$is replaced by $\mathrm{NH}_{4}^{+}$, only occurred when $\mathrm{SO}_{4}^{2-}$ was the main anion in the medium. With $\mathrm{Cl}^{-}$, $\mathrm{NH}_{4}^{+}$authorized the same growth as $\mathrm{NO}_{3}^{-}$. In the later case, more than half of the anion accumulation was accounted for by organic acids (estimated as the difference between the accumulations of main exogenous cations and anions). When $\mathrm{NH}_{4}^{+}$was the $\mathrm{N}$ source, similar amounts of exogenous anions and cations were accumulated. However, the global ion accumulation was higher when $\mathrm{Cl}^{-}$absorption was possible, than when only $\mathrm{SO}_{4}^{2-}$ and $\mathrm{H}_{2} \mathrm{PO}_{4}^{-}$were available in the medium. Thus, it seems that the growth reduction observed in the later case was due to the inability of the plants to accumulate exogenous anions, while net synthesis of organic anion was impedded by $\mathrm{NH}_{4}^{+}$assimilation.

barley - nitrate - ion accumulation - growth - $\mathrm{H}+$ transport

\section{INTRODUCTION}

Bien que $\mathrm{NO}_{3}^{-}$soit en général leur source principale d'azote, les plantes cultivées utilisent aussi $\mathrm{NH}_{4}^{+}$. Cette dernière source devient prépondérante lorsque les conditions du sol sont incompatibles avec une bonne nitrification (Wallace et al., 1978; Mc Clung \& Brankenberger, 1987). La plupart des comparaisons des 2 sources de $\mathrm{N}$ ont indiqué que la croissance sur $\mathrm{NO}_{3}^{-}$est meilleure que sur $\mathrm{NH}_{4}^{+}$(Woolhouse \& Hardwick, 1966; Kirkby \& Mengel, 1967; Chaillou et al., 1986). Ces résultats paraissent paradoxaux, car l'utilisation de $\mathrm{NH}_{4}^{+}$est théoriquement moins coûteuse en énergie que celle de $\mathrm{NO}_{3}^{-}$. D'une part, elle courtcircuite les étapes de réduction catalysées par la nitrate réductase et la nitrite réductase. Ainsi, le coût énergétique de l'assimilation de $\mathrm{NH}_{4}^{+}$est 3 fois moindre que celui de $\mathrm{NO}_{3}^{-}$, lorsqu'on le calcule à partir des nombres d'ATP et de cofacteurs réduits utilisés (Salsac et al., 1987). D'autre part, l'assimilation de l'anion $\mathrm{NO}_{3}^{-}$et celle du cation $\mathrm{NH}_{4}^{+}$entraînent des perturbations opposées de l'équilibre acido-basique des cellules. La première libère des équivalents $\mathrm{OH}^{-}$, et la seconde des équivalents $\mathrm{H}^{+}$. Ces perturbations sont corrigées par 2 sortes de mécanismes, qui peuvent éventuellement agir parallèlement : des excrétions de 
$\mathrm{H}^{+}$ou $\mathrm{OH}^{-}$, et des synthèses d'ions organiques (Raven, 1985a). Lorsqu'on comptabilise les coûts énergétiques de ces divers mécanismes concourant à maintenir l'équilibre acido-basique des cellules, on trouve qu'ils sont 3 fois plus élevés dans le cas de l'assimilation de $\mathrm{NO}_{3}^{-}$que dans celui de l'assimilation de $\mathrm{NH}_{4}^{+}$(Raven, 1985b).

Plusieurs causes de la restriction de la croissance sur $\mathrm{NH}_{4}^{+}$ont été proposées. Cox \& Reisenauer (1973) l'attribuent en partie à l'acidification du milieu qui accompagne l'absorption de $\mathrm{NH}_{4}^{+}$et limite cette dernière (Bennett \& Adams, 1970). Effectivement, si le pH du milieu est contrôlé, $\mathrm{NH}_{4}^{+}$est mieux absorbé que $\mathrm{NO}_{3}^{-}$(Coic et al., 1961; Barker et al., 1966; Aslam, 1984, Troelstra et al., 1985). Une autre explication de la croissance limitée sur $\mathrm{NH}_{4}^{+}$est tirée des études des compositions ioniques des tissus. L'utilisation de $\mathrm{NH}_{4}^{+}$comme source d'azote entraîne (par rapport à l'utilisation de $\mathrm{NO}_{3}^{-}$) une réduction des teneurs en cations (Blair et al., 1970) et une augmentation des teneurs en anions minéraux autres que $\mathrm{NO}_{3}^{-}$(Asher \& Loneragan, 1967), accompagnée d'une baisse de l'accumulation des anions organiques. Ce défaut de production d'anions organiques est considéré par Salsac et al. (1987) comme un facteur probable de la réduction de croissance sur $\mathrm{NH}_{4}^{+}$. En effet, seules les espèces capables de produire ces acides indépendamment de l'assimilation de $\mathrm{NO}_{3}^{-}$sont capables d'utiliser $\mathrm{NH}_{4}^{+}$avec autant d'efficacité que $\mathrm{NO}_{3}^{-}$.

Le travail présenté ici vise à montrer que c'est la capacité d'accumulation anionique (indépendamment du type d'anion, minéral ou organique), qui limite la possibilité d'utiliser $\mathrm{NH}_{4}^{+}$. A de rares exceptions près (Dijkshoorn et al., 1968; Lewis et al., 1982), les auteurs ont étudié la nutrition ammoniacale en accompagnant $\mathrm{NH}_{4}^{+}$d'un anion peu perméant. Etant donné que dans les milieux habituels, la concentration de la molécule source de $\mathrm{N}$ est relativement élevée, l'anion accompagnateur de $\mathrm{NH}_{4}^{+}$est l'anion dominant. Or, l'anion utilisé dans la majorité des expériences est $\mathrm{SO}_{4}^{2-}$, peu perméant. Nous avons comparé les effets de $\mathrm{NO}_{3}^{-}$et $\mathrm{NH}_{4}^{+}$sur la croissance et la nutrition de l'orge, selon que l'anion dominant du milieu est $\mathrm{SO}_{4}^{2-}$, ou $\mathrm{Cl}^{-}$, très perméant chez cette plante.

\section{MATÉRIELS ET MÉTHODES}

\section{Conduite des cultures}

L'orge Martin est une variété d'Hordeum vulgare L. largement cultivée en Tunisie; l'orge Qerqena est un isolat originaire des lles Qerqena (Tunisie), où il est souvent cultivé dans des sols salés. Après germination sur papier filtre humide, les plantules sont transférées sur des claies de plastique au-dessus de pots de
$750 \mathrm{ml}$ remplis d'eau distillée. Au stade première feuille (5 à $6 \mathrm{j}$ après la germination), l'eau est remplacée par un milieu nutritif, où l'azote est apporté sous forme $\mathrm{NO}_{3}^{-}$ou $\mathrm{NH}_{4}^{+}$. Un premier prélèvement de plantes est fait à l'apparition de la seconde feuille $\left(12^{\circ}\right.$ ou $13^{\circ} \mathrm{j}$ ). Le prélèvement final a lieu 23 j plus tard. La durée de la culture couvre donc la quasi totalité du pic d'absorption (quantités d'ions absorbées par jour et par $\mathrm{g}$ de racine), qui se situe entre les $18^{\circ}$ et $40^{\circ} j$ après le semis chez plusieurs cultivars d'orge (Perby \& Jensen, 1983).

Les compositions des milieux en macroéléments sont données dans le Tableau I. Les oligoéléments et le fer sont introduits respectivement selon Arnon \& Hoagland (1940) et Jacobson (1951). Dans certains cas, $\mathrm{CaCO}_{3}(2$ g.l-1) est ajouté aux milieux, afin d'éviter l'acidification due à l'assimilation de $\mathrm{NH}_{4}^{+}$(Lewis et al., 1986). Les milieux sont aérés par bullage d'air, et renouvelés tous les $4 \mathrm{j}$. Cette périodicité, choisie à l'aide d'expériences préliminaires, permet de limiter l'épuisement du volume de solution dans chaque pot à moins de $10 \%$. La photopériode est de $14 \mathrm{~h}$, avec un rayonnement efficace de $400 \mu$ mole.m-2.$s^{-1}$. L'humidité relative et la température sont respectivement $65 \pm 5 \%$ et $25 \pm 3^{\circ} \mathrm{C}$ pendant la photopériode, et $80 \pm 5 \%$ et $18 \pm 3 \% \mathrm{C}$ pendant la nyctipériode. Avec chaque type d'orge, 2 répétitions totalement indépendantes sont faites pour chaque expérience. Sauf indication contraire, 8 pots sont utilisés dans chaque expérience et chaque traitement, correspondant chacun à 4 plantes pour le premier prélèvement, et 4 autres pour le second prélèvement.

\section{Mesures}

Toutes les mesures de masse et les analyses sont faites sur 4 plantes traitées séparément. Les plantes récoltées sont scindées en parties aériennes et racines, pesées (matière fraîche), séchées à l'étuve, et pesées à nouveau (matière sèche, MS). La croissance moyenne relative (CMR, $\left.j^{-1}\right)$ est calculée par la relation CMR $=\left(M_{2}-M_{1}\right)(1 / M) /\left(t_{2}-t_{1}\right)$, où $M_{1}$ et $M_{2}$ sont les masses de matière sèche aux temps $t_{1}(12 \mathrm{j})$ et $t_{2}(35 j)$, et $M=\left(M_{1}+M_{2}\right) / 2$.

Les échantillons sont ensuite réduits en poudre fine à l'aide d'un broyeur à billes. Les cations sont dosés par photométrie de flamme en émission $\left(\mathrm{K}^{+}, \mathrm{Na}^{+}\right.$, $\left.\mathrm{Ca}^{2+}\right)$ ou en absorption atomique $\left(\mathrm{Mg}^{2+}\right)$, après extraction par $\mathrm{HNO}_{3}^{-} 0,1 \mathrm{M}$. Sur le même extrait, $\mathrm{Cl}^{-}$est dosé par coulométrie, $\mathrm{SO}_{4}^{2-}$ par turbidimétrie à $360 \mathrm{~nm}$, et $\mathrm{PO}_{4}^{3-}$ par colorimétrie en présence d'un réactif nitrovanadomolybdique. L'azote total est obtenu par distillation et titrimétrie après minéralisation à chaud de la poudre sèche $\left(\mathrm{H}_{2} \mathrm{SO}_{4}\right.$ concentré, poudre de $\mathrm{Zn}$ et catalyseur au sélenium). L'azote protéique est déterminé par la même procédure, appliquée au précipité obtenu par traitement de la poudre sèche avec l'acide trichloroacétique $(10 \%)$. $\mathrm{NO}_{3}^{-}$est dosé par colorimétrie sur l'extrait aqueux (Jackson 1958).

Les consommations d'eau sont évaluées en mesurant la diminution du volume de milieu entre 2 renouvellements, et en la corrigeant par l'évaporation mesurée avec des pots sans plante. Les échanges nets d'équivalents $\mathrm{H}^{+} / \mathrm{OH}^{-}$sont déterminés par titrations acidobasiques des milieux usagés. 
Tableau I. Concentrations des macroéléments dans les solutions nutritives. Les milieux diffèrent par la source azotée, et par la nature de l'anion dominant dans le cas des milieux $\mathrm{NH}_{4}^{+}\left(\mathrm{Cl}^{-}\right.$ou $\left.\mathrm{SO}_{4}^{2}\right)$. Les milieux $\mathrm{NO}_{3}^{-}$de types 1 et 2 sont respectivement utilisés comme témoins pour les expériences avec le milieux $\mathrm{NH}_{4}^{+}$de types $1\left(\mathrm{Cl}^{-}\right)$et $2\left(\mathrm{SO}_{4}^{2-}\right)$.

\begin{tabular}{|c|c|c|c|c|c|c|c|c|c|c|c|}
\hline \multirow{2}{*}{ Milieux } & \multicolumn{10}{|c|}{ Concentrations $(\mathrm{mN})$} & \multirow{2}{*}{$\begin{array}{c}\text { Osmolarité } \\
\text { calculée } \\
\text { (mM) }\end{array}$} \\
\hline & $K^{+}$ & $\mathrm{Ca}^{2+}$ & & $M g^{2+}$ & $\mathrm{NH}_{4}^{+}$ & & & $\mathrm{H}_{2} \mathrm{PO}_{4}^{-}$ & $\mathrm{SO}_{4}^{2-}$ & $C r$ & \\
\hline $\mathrm{NO}_{3}^{-}$type 1 & 2,2 & 4,0 & 1,5 & 0,0 & 4,0 & 2,2 & 1,5 & 0,0 & 11,9 & & \\
\hline $\mathrm{NH}_{4}^{+}$type 1 & 2,2 & 4,0 & 1,5 & 4,0 & 0,0 & 2,2 & 1,5 & 8,0 & 19,9 & & \\
\hline $\mathrm{NO}_{3}^{-}$type 2 & 2,6 & 3,4 & 1,4 & 0,0 & 5,0 & 1,0 & 1,4 & 0,0 & 11,7 & & \\
\hline $\mathrm{NH}_{4}^{+}$type 2 & 2,6 & 1,7 & 1,4 & 5,0 & 0,0 & 1,0 & 9,7 & 0,0 & 15,0 & & \\
\hline
\end{tabular}

\section{RESULTATS}

\section{Croissance}

Les effets de la source azotée $\left(\mathrm{NO}_{3}^{-}\right.$ou $\left.\mathrm{NH}_{4}^{+}\right)$et de l'anion dominant en milieu ammoniacal $\left(\mathrm{Cl}^{-}\right.$ou $\mathrm{SO}_{4}^{2-}$ ) sur les quantités de matière sèche à la récolte finale sont illustrés par la Figure 1 . Comparé au milieu $\mathrm{NO}_{3}^{-}$, le milieu $\mathrm{NH}_{4}^{+}$induit une croissance accrue en présence de $\mathrm{Cl}^{-}$, diminuée en présence de $\mathrm{SO}_{4}^{2-}$. Ces effets apparaissent encore plus nettement lorsqu'on compare les vitesses de croissance moyennes relatives (entre $12 \mathrm{j}$ et $35 \mathrm{j}$ ) obtenues sur les différents milieux (Fig. 2).

Pendant les 4 j qui séparent 2 renouvellements de milieu, le $\mathrm{pH}$ des solutions $\mathrm{NH}_{4}^{+}$baisse

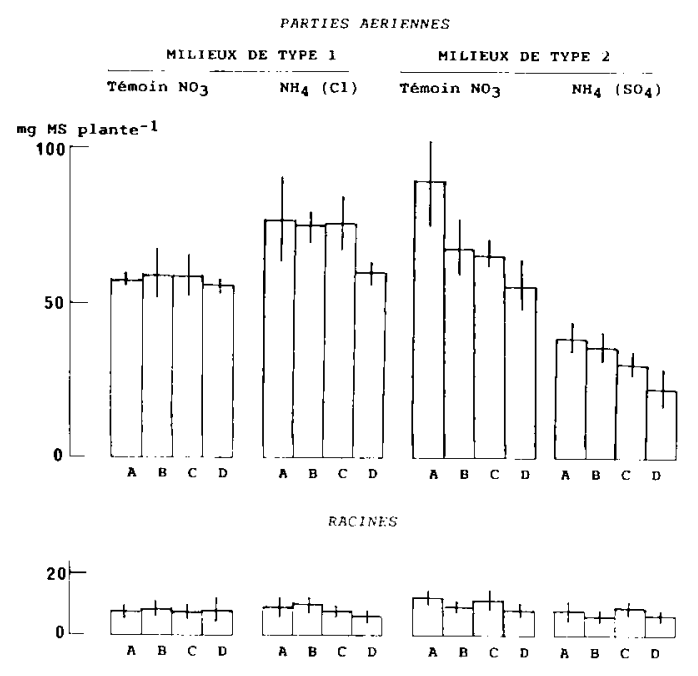

Fig. 1. Effets de la source d'azote et de l'anion dominant sur la croissance. Les histogrammes représentent les masses de matière sèche à $35 \mathrm{j}$. En haut : parties aériennes; en bas : racines. A et $B: 2$ expériences avec l'orge Martin; $C$ et $D: 2$ expériences avec l'orge Qerqena (moyenne de 4 pots contenant chacun 8 plantes pour chaque expérience). Les barres verticales sont les intervalles de confiance au seuil de $95 \%$. Les noms des milieux portés au-dessus des histogrammes font référence aux sources d'azote et aux anions dominants (Tableau I). de 6,2 (solution de type $1: \mathrm{Cl}^{-}$comme anion majeur), ou de 5,4 (type $2: \mathrm{SO}_{4}^{2-}$ ), jusqu'à 3 . Le $\mathrm{pH}$ des solutions $\mathrm{NO}_{3}^{-}$de types 1 et 2 augmente de 6,2 et 5,4 jusqu'à 6,7 et 6,8 . Pour apprécier l'effet de ces diminutions du $\mathrm{pH}$ en milieu $\mathrm{NH}_{4}^{+}$, nous avons reproduit les expériences avec l'orge Martin, en ajoutant $\mathrm{CaCO}_{3}$ à tous les milieux. Les $\mathrm{pH}$ des 4 milieux neufs sont alors les mêmes $(7,5)$. Les valeurs extrêmes dans les milieux usagés sont 7,8 et 7,9 pour les milieux $\mathrm{NO}_{3}^{-}$de types 1 et 2, et 7,4 pour les 2 milieux $\mathrm{NH}_{4}^{+}$. Le Tableau II montre que dans tous les cas, la croissance est stimulée par l'addition de $\mathrm{CaCO}_{3}$. Cette addition atténue les différences entre les effets des sources d'azote, et les effets des anions dominants (sauf en ce qui concerne le développement important des racines sur le milieu $\mathrm{NO}_{3}^{-}$de type 1); mais en l'absence de carbonate, c'est encore $\mathrm{NH}_{4}^{+}$accompagné de $\mathrm{SO}_{4}^{2-}$ qui entraîne la croissance la plus faible. Dans la suite du texte, les résultats exposés sont obtenus en absence de $\mathrm{CaCO}_{3}$.

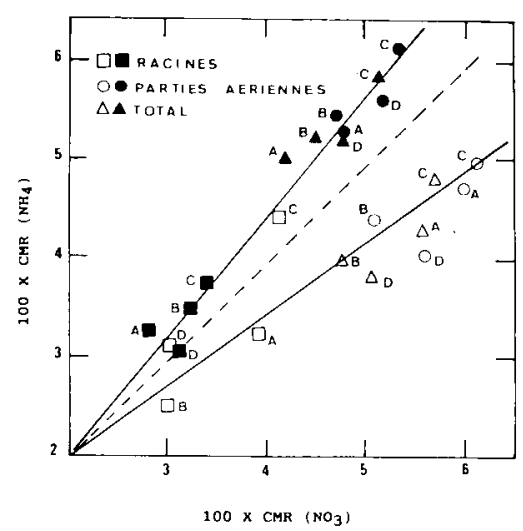

Fig. 2 Comparaison des effets de la source d'azote et de l'anion dominant sur la vitesse moyenne de croissance relative $\left(C M R, j^{-1}\right)$. A, B, C et D : comme sur la Figure 1. Abscisses : culture sur $\mathrm{NO}_{3}^{-}$; ordonnées : sur $\mathrm{NH}_{4}^{+}$. Symboles pleins : milieux de type 1 (Tableau I; $\mathrm{NH}_{4}^{+}$accompagné de $\left.\mathrm{Cl}^{-}\right)$. Symboles ouverts : milieux de type $2\left(\mathrm{NH}_{4}^{+}\right.$accompagné de $\left.\mathrm{SO}_{4}^{2-}\right)$. Les 2 lignes en trait continu sont les droites de régression pour les milieux de types 1 et 2 ; la ligne en tirets est la diagonale. 
Tableau II. Effets de l'addition de $\mathrm{CaCO}_{3}$ aux milieux sur la croissance de l'orge Martin. Les valeurs sont les masses de matière sèche (mg par plante), mesurées à la récolte finale (35 j). Les plantes étaient cultivées depuis $23 \mathrm{j}$ sur les milieux indiqués. Moyennes de 6 pots contenant chacun 7 plantes, et intervalles de confiance au seuil de $95 \%$. Les désignations des différents milieux font référence à la source de $\mathrm{N}\left(\mathrm{NO}_{3}^{-}\right.$et $\left.\mathrm{NH}_{4}^{+}\right)$et à l'anion qui accompagne $\mathrm{NH}_{4}^{+}\left(\mathrm{Cl}^{-}\right.$ et $\left.\mathrm{SO}_{4}^{2-}\right)$ ).

\begin{tabular}{|c|c|c|c|c|c|c|c|c|}
\hline \multirow{3}{*}{ Organes } & \multicolumn{4}{|c|}{ Milieux sans $\mathrm{CaCO}_{3}$} & \multicolumn{4}{|c|}{ Milieux avec $\mathrm{CaCO}_{3}$} \\
\hline & \multicolumn{2}{|c|}{ Type 1} & \multicolumn{2}{|r|}{ Type 2} & \multicolumn{2}{|r|}{ Type 1} & \multicolumn{2}{|c|}{ Type 2} \\
\hline & $\mathrm{NO}_{3}^{-}$ & $\mathrm{NH}_{4}^{+}\left(\mathrm{Cl}^{-}\right)$ & $\mathrm{NO}_{3}^{-}$ & $\mathrm{NH}_{4}^{+}\left(\mathrm{SO}_{4}^{2-}\right)$ & $\mathrm{NO}_{3}^{-}$ & $\mathrm{NH}_{4}^{+} \quad\left(\mathrm{Cl}^{-}\right)$ & $\mathrm{NO}_{3}^{-}$ & $\mathrm{NH}_{4}^{+} \quad\left(\mathrm{SO}_{4}^{2-}\right)$ \\
\hline $\begin{array}{l}\text { PA } \\
\text { R }\end{array}$ & $\begin{array}{r}118 \pm 8 \\
26 \pm 5\end{array}$ & $\begin{array}{l}96 \pm 4 \\
18 \pm 2\end{array}$ & $\begin{array}{r}132 \pm 20 \\
27 \pm 4\end{array}$ & $\begin{array}{l}62 \pm 9 \\
16 \pm 3\end{array}$ & $\begin{array}{r}149 \pm 24 \\
91 \pm 14\end{array}$ & $\begin{array}{r}154 \pm 23 \\
39 \pm 7\end{array}$ & $\begin{array}{r}139 \pm 16 \\
41 \pm 7\end{array}$ & $\begin{array}{r}132 \pm 23 \\
32 \pm 6\end{array}$ \\
\hline
\end{tabular}

PA : parties aériennes

$R$ : racines

\section{Economie d'eau}

Comparé au traitement $\mathrm{NO}_{3}^{-}$, le traitement $\mathrm{NH}_{4}^{+}$ de type $1\left(\mathrm{Cl}^{-}\right)$n'affecte pas la teneur en eau des feuilles à la récolte finale, mais il diminue la quantité d'eau consommée par les plantes entre $12 \mathrm{j}$ et $35 \mathrm{j}$ (Tableau III). II en résulte que l'efficience de l'utilisation de l'eau pour la croissance (masse de MS produite par I d'eau utilisée) est augmentée. Par contre, lorsque $\mathrm{NH}_{4}^{+}$est accompagnée de $\mathrm{SO}_{4}^{2-}$, la teneur en eau des feuilles est abaissée, sans que l'efficience varie significativement. Dans ce cas, la diminution de la consom- mation d'eau n'est due qu'à la baisse de croissance.

\section{Assimilation de l'azote}

La Figure 3 permet de comparer les effets de la source d'azote et de l'anion dominant sur les teneurs en azote réduit total ou en azote protéique, à la récolte finale. Cette représentation montre que ces 2 teneurs sont en général peu dépendantes de la nature des milieux. Ceci suggère que les effets des différents traitements sur la croissance ne s'exercent pas par l'intermédiai-

Tableau IIl. Effets de la source d'azote et de l'anion dominant sur les paramètres hydriques. Les plantes, âgées de 35 j à la récolte, sont cultivées depuis $23 \mathrm{j}$ sur les milieux indiqués (cf Tableau I). A, B, C, D : comme sur la Figure 1 . Moyennes de 4 pots contenant chacun 8 plantes pour chaque expérience. Teneur : teneur en eau des feuilles (ml.g ${ }^{-1}$ MS); consommation : quantité moyenne d'eau utilisée ( $\mathrm{ml} . \mathrm{j}^{-1}$ pour 100 plantes); efficience : efficience de l'utilisation de l'eau pour la croissance, g MS produit par I d'eau consommé (plante entière).

Source d'azote : $\mathrm{NO}_{3}^{-}$

Paramètres
$A$

$B$

$C$

$D$
Source d'azote : $\mathrm{NH}_{4}^{+}$

\begin{tabular}{|c|c|c|c|c|c|c|c|c|}
\hline \multicolumn{9}{|c|}{ Milieux de type $1\left(\mathrm{NH}_{4}^{+}\right.$accompagné par $\left.\mathrm{Cl}^{-}\right)$} \\
\hline $\begin{array}{l}\text { Teneur } \\
\text { Consommation }\end{array}$ & $\begin{array}{l}7,7 \pm 0,4 \\
387 \pm 9\end{array}$ & $\begin{array}{c}8,3 \pm 0,3 \\
243\end{array}$ & $\begin{array}{c}8,3 \pm 0,7 \\
383 \pm 144\end{array}$ & $\begin{array}{c}10,1 \pm 0,8 \\
244\end{array}$ & $\begin{array}{c}8,6 \pm 0,3 \\
274 \pm 52\end{array}$ & $\begin{array}{c}9,7 \pm 0,6 \\
181\end{array}$ & $\begin{array}{c}8,6 \pm 0,2 \\
291 \pm 87\end{array}$ & $\begin{array}{c}10,3 \pm 0,3 \\
175\end{array}$ \\
\hline Efficience & $0,74 \pm 0,05$ & 1,13 & $0,77 \pm 0,11$ & 1,22 & $1,36 \pm 0,24$ & 2,05 & $1,28 \pm 0,30$ & 1,56 \\
\hline
\end{tabular}

Milieux de type $2\left(\mathrm{NH}_{4}^{+}\right.$accompagné par $\left.\mathrm{SO}_{4}^{2-}\right)$

$\begin{array}{lcccccccc}\text { Teneur } & 9,3 \pm 0,3 & 9,4 \pm 0,3 & 10,8 \pm 0,6 & 11,5 \pm 0,5 & 6,0 \pm 0,6 & 6,6 \pm 0,1 & 6,7 \pm 0,7 & 7,7 \pm 0,5 \\ \text { Consommation } & 276 \pm 16 & 248 \pm 61 & 213 \pm 9 & 204 \pm 22 & 162 \pm 20 & 130 \pm 22 & 142 \pm 8 & 109 \pm 35\end{array}$




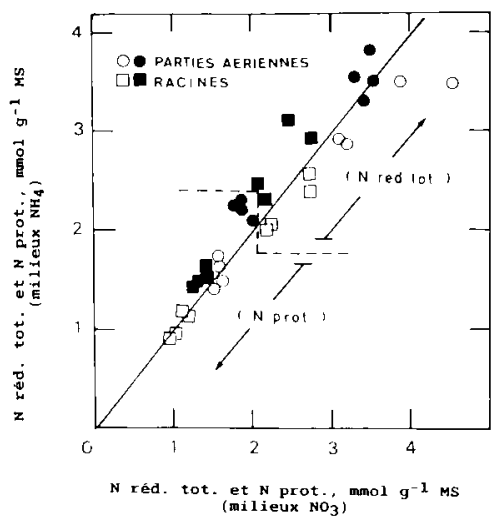

Fig. 3. Comparaison des effets de la source d'azote et de l'anion dominant sur les accumulations de $\mathrm{N}$ réduit et de $\mathrm{N}$ protéique. Chaque point met en relation les teneurs mesurées sur des plantes âgées de $35 \mathrm{j}$, cultivées sur des milieux différant par la source d'azote $\left(\mathrm{NO}_{3}^{-}\right.$et $\left.\mathrm{NH}_{4}^{+}\right)$. Symboles pleins : milieux de type 1 (Tableau I; $\mathrm{NH}_{4}^{+}$accompagné de $\mathrm{Cl}$ ). Symboles ouverts : milieux de type $2\left(\mathrm{NH}_{4}^{+}\right.$accompagné de $\left.\mathrm{SO}_{4}^{2-}\right)$. Pour chaque traitement, les 4 points correspondent à 2 expériences avec l'orge Martin et deux expériences avec l'orge Qerqena (moyenne de 4 pots contenant chacun 8 plantes pour chaque expérience). La droite est la diagonale.

re de perturbations de l'assimilation de l'azote ou de la synthèse protéique.

\section{Equilibres ioniques}

Les concentrations équivalentaires globales des cations et des anions dans les parties aériennes sont comparées sur la Figure 4. Dans ces calculs, la valence moyenne du phosphate est prise égale à $-1,5$ (ce qui revient à supposer que le $\mathrm{pH}$ moyen est proche de la neutralité). Cette approximation repose sur le fait que le phosphate est réparti entre le cytoplasme $(\mathrm{pH}$ supérieur à 7) et la vacuole (acide). L'écart entre la somme des cations $\mathrm{C}^{+}$et celle des anions $\mathrm{A}^{-}$ représente une estimation des ions organiques $\mathrm{R}^{-}$ou $\mathrm{R}^{+}$. En présence de $\mathrm{NO}_{3}^{-}$, il y a toujours un excès important de $\mathrm{C}^{+}$sur $\mathrm{A}^{-}$, c'est-à-dire une accumulation de $\mathrm{R}^{-}$. Au contraire, sur milieu $\mathrm{NH}_{4}^{+}$, les quantités de $C^{+}$et $A^{-}$sont relativement proches (avec parfois une légère accumulation de $\mathrm{R}^{+}$). Cela correspond au remplacement de l'accumulation des anions organiques par une accumulation d'anions minéraux, surtout $\mathrm{Cl}^{-}$ou $\mathrm{SO}_{4}^{2-}$.

\section{Transports d'équivalents $\mathrm{H}^{+}$et assimilation de $N$}

Les quantités d'azote assimilé sont estimées par différence entre les quantités de $N$ réduit par plante à $12 \mathrm{j}$ et à $35 \mathrm{j}$. Le Tableau IV rassemble les valeurs obtenues, ainsi que les valeurs des échanges de $\mathrm{H}^{+}$entre la plante et le milieu pour

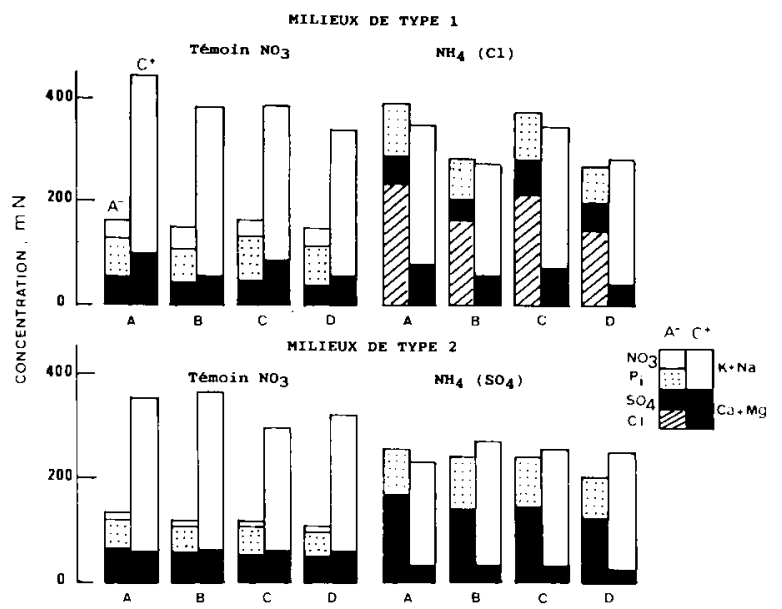

Fig. 4. Effets de la source d'azote et de l'anion dominant sur l'accumulation ionique dans les parties aériennes. Les concentrations moyennes sont obtenues en divisant les quantités d'ions ( $\mu \mathrm{eq})$ par les quantités d'eau $(\mathrm{ml})$ dans les tissus. Les plantes sont âgées de $35 \mathrm{j}$ à la récolte. Les noms des milieux au-dessus des histogrammes font référence aux sources d'azote et aux anions dominants (ct. Tableau I). A, B, $C$ et $D$ : comme sur la Figure 1.

la période $12 \mathrm{j}-35 \mathrm{j}$. Elles sont obtenues par des titrations des milieux à chaque renouvellement. On observe des absorptions nettes d'équivalents $\mathrm{H}^{+}$(ou exsorptions de $\mathrm{OH}^{-}$) quand la source de $\mathrm{N}$ est $\mathrm{NO}_{3}^{-}$, et des exsorptions nettes d'équivalents $\mathrm{H}^{+}$dans le cas de $\mathrm{NH}_{4}^{+}$. Les rapports (quantité de $\mathrm{N}$ réduit)/(quantité de $\mathrm{H}^{+}$échangé) sont $0,46 \pm 0,11$ et $0,45 \pm 0,16$ pour les 2 milieux $\mathrm{NO}_{3}^{-}$et $0,97 \pm 0,22$ et $1,17 \pm 0,37$ pour les milieux $\mathrm{NH}_{4}^{+}$ contenant respectivement $\mathrm{Cl}^{-}$et $\mathrm{SO}_{4}^{2-}$ comme anions majeurs. Compte tenu de l'imprécision des mesures des flux nets de $\mathrm{H}^{+}$sur une longue période, on peut considérer que $0,5 \mathrm{~mole}^{-}$ est excrétée par mole de $\mathrm{NO}_{3}^{-}$, et $1 \mathrm{~mole}^{+}$est excrétée par mole $\mathrm{NH}_{4}^{+}$assimilé.

\section{Accumulation des osmoticums}

L'estimation de la part des ions dans l'osmolarité des tissus est faite à partir des concentrations moyennes dans les parties aériennes (Fig. 4). Ceci nécessite plusieurs hypothèses. On admet que, parmi les cations, $\mathrm{Ca}^{2+}$ et $\mathrm{Mg}^{2+}$ sont en grande partie liés à des macromolécules, et ne participent pas au potentiel osmotique. Tous les anions sont comptés comme osmotiquement actifs. Lorsque le bilan ionique est équilibré par $R^{-}$(Fig. 4), on calcule la molarité de ces acides en leur attribuant une valence moyenne de $-1,5$. Chez l'orge, la forme dominante de $\mathrm{R}^{-}$est le malate, accumulé dans la vacuole (Jacobson, 1955; Jacoby \& Laties, 1971). Les pK de l'acide malique sont 3,4 et 5,2 : la valence moyenne $-1,5$ correspond donc à un $\mathrm{pH}$ vacuolaire de 5,2 , 
Tableau IV. Relation entre l'assimilation de $\mathrm{N}$ et les échanges d'équivalents $\mathrm{H}^{+}$. L'assimilation d'azote $\left(\mathrm{J}_{\text {Nass }}\right)$ est estimée par la différence entre les quantités de $\mathrm{N}$ réduit accumulées à $12 \mathrm{j}$ et $35 \mathrm{j}$ (voir le texte). L'échange net de $\mathrm{H}+$ sur cette période $\left(\mathrm{J}_{\mathrm{H}_{+}}\right)$est déterminé par titration des milieux de culture à chaque renouvellement (valeurs négatives : efflux net). Toutes les valeurs sont exprimées en $\mu$ eq.j-j pour 100 plantes. A, B, C, D : comme sur la Figure 1. Moyennes de 4 pots contenant chacun 8 plantes pour chaque expérience.

\begin{tabular}{|c|c|c|c|c|c|c|c|c|}
\hline \multirow{2}{*}{$\begin{array}{l}\text { Flux nets } \\
\text { moyens }\end{array}$} & \multicolumn{4}{|c|}{ Source d'azote : $\mathrm{NO}_{3}^{-}$} & \multicolumn{4}{|c|}{ Source d'azote : $\mathrm{NH}_{4}^{+}$} \\
\hline & $A$ & $B$ & $c$ & $D$ & $A$ & $B$ & $c$ & $D$ \\
\hline
\end{tabular}

Milieux de type $1\left(\mathrm{NH}_{4}^{+}\right.$accompagné par $\left.\mathrm{Cl}-\right)$

\begin{tabular}{|c|c|c|c|c|c|c|c|c|}
\hline$J C$ & 1032 & 907 & 921 & 924 & 2528 & 2215 & 2294 & 1697 \\
\hline $\mathrm{J}_{\mathrm{A}}$ & 1278 & 1208 & 1395 & 1229 & 1343 & 1041 & 1146 & 823 \\
\hline$J_{C}-J_{A}$ & -246 & -301 & -474 & -305 & 1185 & 1174 & 1148 & 874 \\
\hline$J_{\text {Nass }}$ & 898 & 817 & 993 & 817 & 1390 & 1189 & 1219 & 846 \\
\hline $\mathrm{J}_{\mathrm{H}^{+}}$ & 404 & 438 & 409 & 406 & -1196 & -1359 & -1035 & -860 \\
\hline
\end{tabular}

Milieux de type $2\left(\mathrm{NH}_{4}^{+}\right.$accompagné par $\left.\mathrm{SO}_{4}^{2-}\right)$

\begin{tabular}{lrrrrrrrr}
$J_{\mathrm{C}}$ & 2019 & 1255 & 1485 & 1021 & 1242 & 881 & 1059 & 655 \\
$J_{\mathrm{A}}$ & 2359 & 1680 & 1786 & 1624 & 510 & 374 & 401 & 286 \\
$\mathrm{~J}_{\mathrm{C}} \mathrm{J}_{\mathrm{A}}$ & -340 & -425 & -301 & -603 & 732 & 507 & 658 & 369 \\
$\mathrm{~J}_{\text {Nass }}$ & 1636 & 1180 & 1224 & 1185 & 774 & 578 & 672 & 378 \\
$\mathrm{~J}_{\mathrm{H}^{+}}$ & 863 & 450 & 660 & 414 & -935 & -701 & -911 & -483 \\
\hline
\end{tabular}

valeur raisonnable. Les composés azotés réduits solubles (acides aminés, amides, etc.) sont estimés par différence entre l'azote total réduit et l'azote protéique, en comptant 1 osmol pour 1 mole $N$. Enfin, lorsque les cations organiques $R^{+}$ sont présents $\left(A^{-}>C^{+}\right)$, on admet qu'ils sont monovalents et déjà décomptés dans les composés azotés réduits solubles. La Figure 5 montre les résultats de ces estimations. L'osmolarité ainsi calculée est sensiblement la même pour les

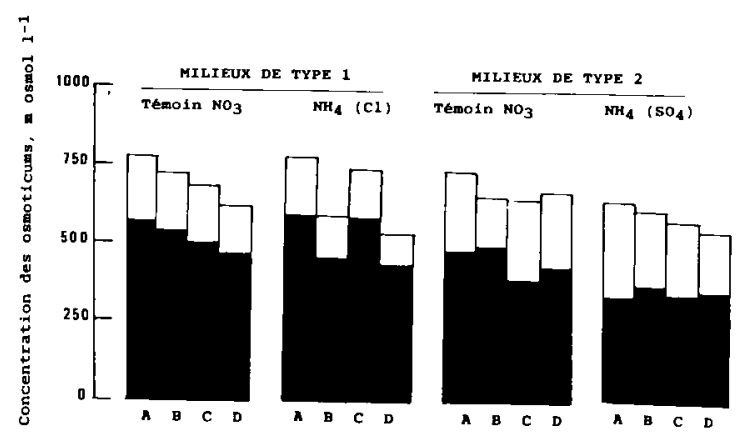

Fig. 5. Effets de la source d'azote et de l'anion dominant sur l'accumulation des osmoticums. Les histogrammes donnent les estimations de l'osmolarité moyenne dans les parties aériennes revenant aux ions minéraux et organiques (en noir), et aux composés azotés réduits solubles. Ces estimations sont obtenues à partir des données de la Figure 4 , et des dosages d'azote réduit soluble (voir le texte). Les plantes sont âgées de $35 \mathrm{j}$ à la récolte. Les noms des milieux au-dessus des histogrammes font référence aux sources d'azote et aux anions dominants ( $c f$ Tableau I). A, B, C et D : comme sur la Figure 1. plantes cultivées en présence de $\mathrm{NO}_{3}^{-}$et $\mathrm{NH}_{4}^{+}$, quand $\mathrm{Cl}^{-}$est l'anion dominant. Ceci résulte du remplacement, dans les tissus, de $\mathrm{R}^{-}$(milieux $\mathrm{NO}_{3}^{-}$) par $\mathrm{Cl}^{-}$(milieu $\mathrm{NH}_{4}^{+}$) (Fig. 4). Lorsque $\mathrm{NH}_{4}^{+}$ est accompagné $\mathrm{SO}_{4}^{2-}$, l'osmolarité calculée est légèrement plus faible que dans les autres cas. La part due aux ions est abaissée, mais celle des composés azotés est plus importante.

\section{DISCUSSION}

Les effets de la source d'azote et de l'anion dominant ont été étudiés sur 2 variétés, et pour chacune, avec 2 expériences indépendantes de longue durée. La présentation des résultats individuels par répétition et par variété $(A, B, C, D)$ permet d'illustrer la généralité des comportements analysés ci-après. Nos résultats montrent que la substitution de $\mathrm{NH}_{4}^{+}$à $\mathrm{NO}_{3}^{-}$comme source d'azote n'entraîne pas obligatoirement une réduction de la vitesse de croissance. La capacité de la plante à croître rapidement sur milieu $\mathrm{NH}_{4}^{+}$dépend de la présence d'un anion perméant dans le milieu ( $\mathrm{Cl}^{-}$, ici) (Fig. 2). Effectivement, les anions $\mathrm{R}^{-}$, qui constituent la majorité de l'accumulation anionique dans les traitements $\mathrm{NO}_{3}^{-}$, sont quasiment absents quand les plantes sont cultivées sur milieu $\mathrm{NH}_{4}^{+}$, et seule l'accumulation de $\mathrm{Cl}^{-}$permet de restaurer le niveau d'accumula- 
tion anionique (Fig. 4) à une valeur comparable à celle qu'on observe en milieu $\mathrm{NO}_{3}^{-}$. Par rapport au témoin $\mathrm{NO}_{3}^{-}$, le milieu $\mathrm{NH}_{4}^{+}$accompagné de $\mathrm{SO}_{4}^{2-}$ provoque une baisse du niveau d'accumulation anionique de l'ordre de $25 \%$, d'après la Figure 4. Mais, en réalité, la restriction de l'alimentation anionique est beaucoup plus sévère : la quantité d'anions prélevée par chaque plante est diminuée d'environ $75 \%$ (Tableau IV), car le traitement entraîne une diminution de l'ordre de $40 \%$ de la production de biomasse (Fig. 1).

Les estimations des quantités de $\mathbf{R}$ - produites (bilans $J_{C}-J_{A}$; Tableau IV) et des accumulations de $\mathrm{R}^{-}$(Fig. 4) sont indirectes. Néanmoins, il est peu probable qu'elles soient affectées d'erreurs capables de remettre en cause la conclusion précédente. En effet, elles sont corroborées par la comparaison des transports nets d'équivalents $\mathrm{H}^{+}$ et des quantités de $\mathrm{N}$ assimilées (Tableau IV). L'observation d'environ $0,5 \mathrm{OH}^{-}$exsorbé par $\mathrm{NO}_{3}^{-}$assimilé, signifie que la moitié des équivalents $\mathrm{OH}^{-}$libérés par l'assimilation de $\mathrm{NO}_{3}^{-}$sont conservés sous forme de $\mathrm{R}^{-}$. Sur milieu $\mathrm{NH}_{4}^{+}$, (environ $1 \mathrm{H}^{+}$exsorbé par $\mathrm{NH}_{4}^{+}$assimilé), seuls les $\mathrm{H}^{+}$libérés par l'assimilation de $\mathrm{NH}_{4}^{+}$sont exsorbés : la part qui correspondrait éventuellement à une production nette de $\mathrm{H}^{+}$par carboxylation est donc négligeable.

L'accumulation d'anions exogènes semble nécessaire pour que l'osmolarité moyenne dans les tissus et l'hydratation de ces derniers restent semblables à celles des plantes cultivées en présence de $\mathrm{NO}_{3}^{-}$(Fig. 4 et Tableau III). Les résultats du Tableau III confirment également que le défaut d'hydratation des feuilles, observé quand $\mathrm{NH}_{4}^{+}$est accompagné par $\mathrm{SO}_{4}^{2-}$, résulte d'une difficulté de ces dernières à retenir l'eau plutôt que d'une mauvaise alimentation en eau. En effet, la valeur élevée du coût hydrique de la croissance (par rapport au milieu $\mathrm{NO}_{3}^{-}$témoin) indique que les quantités d'eau qui ont traversé les feuilles (à croissance égale) ont été les mêmes sur milieu $\mathrm{NH}_{4}^{+}$et sur milieu $\mathrm{NO}_{3}^{-}$.

Notre conclusion est que la réduction de croissance souvent observée sur milieu $\mathrm{NH}_{4}^{+}$ne provient pas spécifiquement de l'impossibilité de produire et accumuler des anions organiques (Salsac et al., 1987). Elle résulte de la difficulté générale d'accumuler des anions. Dans la plupart des solutions nutritives utilisées par les auteurs cités dans l'Introduction, $\mathrm{NH}_{4}^{+}$était accompagné de $\mathrm{SO}_{4}^{2-}$, anion peu perméant. Dans ce cas, les anions exogènes ne pouvaient pas compenser le déficit d'anions endogènes dû à l'assimilation de $\mathrm{NH}_{4}^{+}$.

\section{RÉFÉRENCES}

Arnon D.I. \& Hoagland D.R. (1940) Crop production in artificial solutions and in soils with special reference to factors affecting yields and absorption of inorganic nutrients. Soil Sci. 50, 463-485

Asher C.J. \& Loneragan J.F. (1967) Response of plants to phosphate concentration in solution culture. I. Growth and phosphorus content. Soil Sci. 103, 225232

Aslam S.M. (1984) Effect of nutrient solution $\mathrm{pH}$ and $\mathrm{N}$ source $\left(\mathrm{NH}_{4} / \mathrm{NO}_{3}\right)$ on the growth and elemental contents of rice plants. Agronomie 4, 361-365

Barker A.V., Volk A.J. \& Jackson W.A. (1966) Root environment acidity as a regulatory factor in ammonium assimilation by the bean plant. Plant Physiol. 41, 1193-1199

Bennett A.C. \& Adams F. (1970) Concentration of $\mathrm{NH}_{3}$ (aq.) required for incipient $\mathrm{NH}_{3}$ toxicity to seedlings. Soil Sci. Soc. Am. Proc. 34, 259-263

Blair G.J., Miller M.H. \& Mitchell W.A. (1970) Nitrate and ammonium sources of nitrogen for corn and their influence on the intake of other ions. Agron. J. 64, 530532

Chaillou S., Morot-Gaudry J.F., Salsac L., Lesaint C. \& Jolivet $\mathrm{E}$. (1986) Compared effects of $\mathrm{NO}_{3}^{-}$or $\mathrm{NH}_{4}^{+}$on growth and metabolism of french bean. Physiol. Vég. 24, 679-687

Coïc Y., Lesaint C. \& Leroux F. (1961) Comparaison de l'influence de la nutrition nitrique et ammoniacale combinée ou non avec une déficience en acide phosphorique, sur l'absorption et le métabolisme des anions et cations, et plus particulièrement des acides organiques chez le maïs. Comparaison du maïs et de la tomate quand à l'effet de la nature et de l'aimentation azotée. Ann. Physiol. Vég. 3, 141-163

Cox W.J. \& Reisenauer H.M. (1973) Growth and ion uptake by wheat supplied with nitrogen as nitrate, or ammonium, or both. Plant Soil 38, 363-380

Dijkshoorn W., Lathwell D.J. \& De Witt C.T. (1968) Temporal changes in carboxylate content of raygrass with stepwise changes in nutrition. Plant Soil 29, 369390

Jackson M.L. (1958) Nitrogen determination for soils and plant tissues. Nitrate determination. In : Soil Chemical Analysis. Prentice Hall Inc., Englewood Cliff, N.J., USA, pp. 181-204

Jacobson L. (1951) Maintenance of iron supply in nutrient solution by a single addition of ferric-potassium-ethylen-diamine-tetracetate. Plant Physiol. 26, 411-413

Jacobson L. (1955) Carbon dioxide fixation and ion absorption in barley roorts. Plant Physiol. 30, 264-269

Jacoby B. \& Laties G.G. (1971) Bicarbonate fixation and malate compartmentation in relation to salt-induced stoichiometric synthesis of organic acid. Plant Physiol. 47, 525-531

Kirkby E.A. \& Mengel K. (1967) lonic balance in different tissues of the tomato plant in relation to nitrate, urea, or ammonium nutrition. Plant Physiol. 46, 6-14

Lewis O.A.M., James D.M. \& Hewitt E.J. (1982) Nitrogen assimilation in barley (Hordeum vulgare $L$. cultivar Mazurka) in response to nitrate and ammonium nutrition. Ann. Bot. 49, 39-49

Lewis O.A.M., Soares M.I.M. \& Lips S.H. (1986) A photosynthetic and $15 \mathrm{~N}$ investigation of the differential 
growth response of barley to nitrate, ammonium, and nitrate + ammonium. In : Fundamental, Ecological, and Agricultural Aspects of Nitrogen Metabolism in Higher Plants (H. Lambers, J.J. Neetson \& I. Stulen eds) Martinus Nijhoff Publishers for the Commission of the European Communities, pp. 295-300

Mc Clung G. \& Frankenberger W.T. Jr. (1987) Nitrogen mineralization rates in saline versus salt-amended soils. Plant Soil, 104, 13-21

Perby H. \& Jensen P. (1983) Varietal differences in uptake and utilization of nitrogen and other macroelements in seedlings of barley, Hordeum vulgare L. Physiol. Plant. 58, 223-230

Raven J.A. (1985a) pH regulation in plants. Sci Progr. Oxford, 69, 495-509

Raven J.A. (1985b) Regulation of $\mathrm{pH}$ and generation of osmolarity in vascular plants. A cost-benefit analysis in relation to efficiency of use of energy, nitrogen, and water. New Phytol. 101, 25-77

Salsac L., Chaillou S., Morot-Gaudry J.F., Lesaint C. \& Jolivet E. (1987) Nitrate and ammonium nutrition in plants. Plant Physiol. Biochem. 25, 805-812

Troelstra S.R. Van Dijk K. \& Blacquière T. (1985) Effect of $\mathrm{N}$ source on proton excretion, ionic balance, and growth of Alnus glutinosa (L.) Goertner : comparison of $\mathrm{N}_{2}$ fixation with single and mixed sources of $\mathrm{NO}_{3}$ and $\mathrm{NH}_{4}$. Plant Soil 84, 361-385

Wallace A., Romney E.M., Kleinkopf G.E. \& Soufi S.M. (1978) Uptake of mineral forms of nitrogen by desert plants. In : Nitrogen in Desert Ecosystems. (West N.E. \& Skuijins J., eds.) Utah State University, IBP Synthesis Series, 9, pp. 130-151

Woolhouse H.W. \& Hardwick M.J. (1966) The growth of tomato seedlings in relation to the form of the nitrogen supply. New Phytol. 65, 518-526 